\title{
An Enhanced MPLS-TE For Transferring Multimedia packets
}

\author{
Abdellah Jamali \\ Dept. of Computer Science and \\ Mathematics \\ ESTB, Hassan 1er University \\ Berrechid, Morocco
}

\author{
Najib Naja \\ Dept. of RIM \\ Institute of Posts and \\ Telecommunications \\ Rabat, Morocco
}

\author{
Driss El Ouadghiri \\ Dept. of Computer Science and \\ Mathematics \\ My Ismail University \\ Meknes, Morocco
}

\begin{abstract}
Multi-Protocol Label Switching is useful in managing multimedia traffic when some links are too congested; MPLS Traffic Engineering is a growing implementation in today's service provider networks. In This paper we propose an improvement of MPLS-TE called EMPLS-TE, it is based on a modification of operation of Forwarding Equivalence Class (FEC) in order to provide the quality of service to stream multimedia. The performance of the EMPLS-TE is evaluated by a simulation model under a variety of network conditions. We also compare its performance with that of unmodified MPLS-TE and MPLS. We demonstrate how a small change to the MPLSTE protocol can lead to significantly improved performance results. We present a comparative analysis between MPLS, MPLS-TE and Enhanced MPLS-TE (EMPLS-TE). Our proposed EMPLS-TE has a performance advantageous for multimedia applications in their movement in a congested and dense environment. EMPLS-TE defines paths for network traffic based on certain quality of service. The simulation study is conducted in this paper; it is a means to illustrate the benefits of using this Enhanced MPLS-TE for multimedia applications.
\end{abstract}

Keywords- Multi-Protocol Label Switching (MPLS); Multi-Protocol Label Switching Traffic Engineering (MPLS-TE); Forwarding Equivalence Class (FEC); Quality Of Service (QoS); Simulation.

\section{INTRODUCTION}

The goal of Traffic Engineering (TE) is to provide QoS to multimedia packets by reservation of the resources and optimum resources utilization [9]. Multiprotocol Label Switching (MPLS) technology [2] allows traffic engineering and enhances the performance of the existing protocols over the traditional IPv4 network. The central idea of MPLS is to attach a short fixed-length label to packets at the ingress router of the MPLS domain. Packet forwarding then depends on the tagged label, not on longest address match, as in traditional IP forwarding. A router placed on the edge of the MPLS domain, named Label Edge Router (LER) that is associated to a label on the basis of a Forwarding Equivalence Class (FEC). In the MPLS network, internal routers that perform swapping and label-based packet forwarding are called Label Switching Routers (LSRs) [15].

MPLS TE also extends the MPLS routing capabilities with support for constraint-based routing. IGPs typically compute routing information using a single metric. Instead of that simple approach, constraint-based routing can take into account more detailed information about network constraints, and policy resources. MPLS TE extends current link-state protocols (IS-IS and OSPF) to distribute such information. There is another approach to provide QoS to multimedia traffic: DiffServ-aware Traffic Engineering (DS-TE) [7] [6], by using three signaling protocols in MPLS networks: Label Distribution Protocol (LDP) [5], Constraint based Routing LDP (CR-LDP) [3] and Resource Reservation Protocol-Traffic Engineering (RSVP-TE) [4].

In this paper we focus on our paper presented in [1] and MPLS-TE as a technology rather used by operators, then we make an improvement on MPLS-TE and propose EMPLS-TE (Enhanced MPLS-TE).

In order to provide a good service for transferring multimedia packets that requires a large flow in the MPLS-TE networks we make an improvement to the method of processing speed in the FEC in MPLS-TE.

\section{Rest of paper is organized as below:}

Section II defines QoS as services that provide some combination of high security, high reliability, low packet drop rate, low delay, and low jitter. The same section reviews the working of traditional IP, MPLS and MPLS-TE and their salient features. In section III, we will describe our proposed enhancement EMPLS-TE and its methods. In section IV, we will simulate the MPLS, MPLS-TE and EMPLS-TE, and then we compare it with original MPLS, and with original MPLSTE. Section V, will conclude this paper. Routing, MPLS and MPLS-TE and their salient features. In section III, we will describe our proposed enhancement EMPLS-TE and its methods. In section IV, we will simulate the MPLS, MPLS-TE and EMPLS-TE, and then we compare it with original MPLS, and with original MPLS-TE. Section V will conclude this paper.

\section{OVERVIEW}

\section{A. Internet Quality of Service (QoS)}

Originally, the Internet was developed for transferring file and accessing remote machines. Therefore, the Internet was not expected to transfer multimedia data at large data rate. Today, many different types of applications in Internet demand more secure more reliable and faster services. Both non-real time and real-time applications require some kinds of 
QoS, such as high reliability, bounded delay and jitter, and high security. Therefore, I would like to define QoS as services that provide some combination of high security, high reliability, low packet drop rate, low delay, and low jitter; in general ATM is an example of a network technology that provides good QoS.

Although ATM can be used to transmit both IP packets and ATM data, it is less suitable for best effort services IP packets mainly because ATM supports only a small part of IP services. The most common and major QoS problem in the backbone network is unevenly distributed traffic. MPLS-TE can distribute traffic evenly and optimize network utilization TE ensures that all available network resources are optimally used during times of failure or traffic routing, which is needed when congestion happens. Network congestion is not easily solved by IP because of its characteristics: connectionless and best effort service. As results, bursts of traffic appear unexpectedly, routers are easily congested, and packets are dropped. Therefore, the current Internet has poor reliability, unbounded delay and jitter, and varied throughput.

\section{B. Traditional IP Routing}

The IP was created as a connectionless network layer protocol that makes no attempt to discriminate between various application types. IP uses routing protocols as traditional Interior Gateway Routing Protocol (IGRP) [10], Intermediate System-to-Intermediate System (IS-IS) [14], Open Shortest Path First (OSPF) [18] to build routing tables for active number the equations consecutively. Equation numbers, within parentheses, are to position flush right, as in (1), using a right tab stop.

Links in an area of network, and therefore transferring data between the source and the destination [16], the operation of these protocols depends on how to promote and distribute information on the state network that are broadcast regularly and depends also on how to update the routing tables of all routers located in the same autonomous system (AS).

Each router uses the information on the overall state of network to maintain an independently its own routing tables so that it can transfer data successfully using the shortest path or the link state as metric maintain before deciding to send data.

The major problem of some of these protocols is that they transfer the data on the paths with minimal hops, and since they do not use the paths with many hops that can lead the data to the destination, then this strategy produces quite congested links.

So, the traditional routing IP traffic is routed by the same types of paths (short), and therefore a fairly large amount of packets is lost.

To tackle the problem of low delay and packet loss during the delivery of multimedia applications, it is necessary to think of improvement methods to use more effectively the available network resources. MPLS and MPLS-TE (MPLS Traffic Engineering) are some process that provides this functionality.

\section{MPLS}

Multiprotocol Label Switching (MPLS) can speed up the flow of network traffic and make it easier to manage. MPLS is flexible, fast, cost-efficient and allows for network segmentation and quality of service (QoS). MPLS also offers a better way of transporting latency-sensitive applications like voice and video. While MPLS technology has been around for several years, businesses are now taking advantage of service provider offerings and beginning their own corporate implementations.

MPLS can be considered a technology that has brought an oriented connection for IP protocol. Therefore, network services and applications can exploit all of the advantages of MPLS. In other words, MPLS is a connection oriented technology that uses a label swapping technique with IP network routing [12]. A label is a small, fixed index, which identifies a Forward Equivalence Class; a group of IP packets that are forwarded over the same path with the same packet treatments. With MPLS, the packet is faster than with use IP address because MPLS uses labels to quickly check the next hop that leads to the destination without going to the network layer to analyze the packets along the path.

MPLS consists of routers: Label Switching Routers (LSR) and Label Edge Routers (LER). These routers use labels to quickly send packets to the destination.

An LSR is a router that forwards both conventional IP packets and MPLS labelled packets. An LER is an LSR at the edge of the MPLS network to add and remove labels. An LER connects between the MPLS domain and the non-MPLS domain such as IP network.

A flow of packets coming from a non-MPLS domain is first assigned a label at an incoming LER and its forward along the path as an old label is replaced with a new label at LSRs on the path. Therefore, a label is used to reach the next node.

Although the exchange of label is required on the path, and the search of the network layer is not required at LSRs routers due to transmission of the link layer with labels. In routers LERS the labels are completely removed and the packets are transmitted directly to other networks. MPLS label switched paths are an essential element in delivering end-to-end QoS. Without them, it is not possible to control the path of packet flows from requested packet treatments.

The assignment of labels to packets is based on the concept of forwarding equivalence class (FEC). According to this concept, packets which belong to the same FEC are assigned the same label at an ingress node to an MPLS domain. A FEC consists of packets entering a network through the same ingress node and exiting the network through the same ingress node. A FEC also consists of packets requiring similar QoS or packet treatment across the MPLS domain. The path traversed by a FEC is called a Label Switched Path (LSP). A signal protocol such as LDP (Load Distribution Protocol) or RSVP (Resource reservation Protocol) [17] is used to establish and release LSPs [13]. 


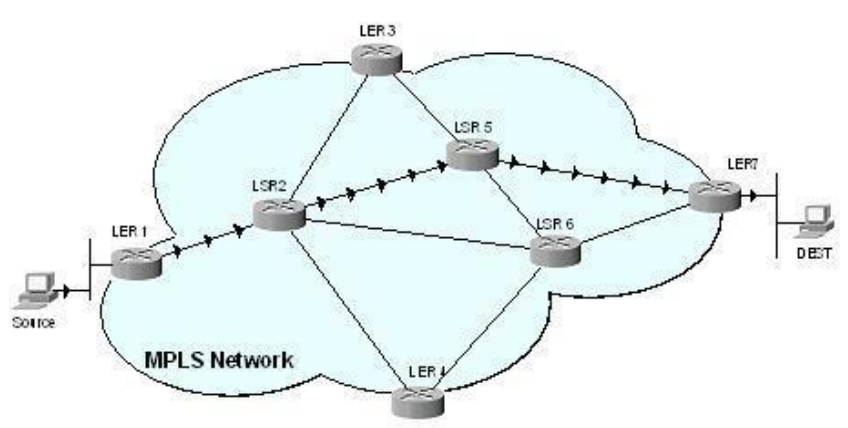

Figure 1. MPLS Network

\section{Traffic Engineering within MPLS}

MPLS Traffic Engineering [11] is an obligation for network operators to provide a fairly reliable infrastructure and provides quality performance. Traffic Engineering provides efficient routing of traffic in the network to the use of network resources. This allows operators the ability to better exploit bandwidth resources on the network [8].

As a result of the unprecedented growth in demand for network resources and the competitiveness amongst providers, Traffic Engineering has become the primary application for MPLS.

MPLS Traffic Engineering responds to the ineffectiveness of some routing protocols in terms of datagram processing in the case of congestion. It allows a wider distribution flow of traffic across all available resources. Load balancing for TE in IP network requires an ability to control traffic flow precisely. In the traditional metric-based control, an administrator can change only link metrics, and the changes of some link metrics may affect the overall traffic flow. To manage the performance of a network, it is necessary to have explicit control over the paths that traffic flows traverse so that traffic flows can be arranged to maximize resource commitments and utilization of the networks [13].

The connection-oriented nature of MPLS allows ISPs to implement TE in their networks and achieve a variety of goals, including bandwidth assurance, different routing, load balancing, path redundancy, and other services that lead to QoS [9].

MPLS networks can use native TE mechanisms to minimize network congestion and improve network performance. TE modifies routing patterns to provide efficient mapping of traffic streams to network resources. This efficient mapping can reduce the occurrence of congestion and improves service quality in terms of the latency, jitter, and loss that packets experience. Historically, IP networks relied on the optimization of underlying network infrastructure or Interior Gateway Protocol (IGP) tuning for TE. Instead, MPLS extends existing IP protocols and makes use of MPLS forwarding capabilities to provide native TE. In addition, MPLS TE can reduce the impact of network failures and increase service availability. RFC 2702 discusses the requirements for TE in MPLS networks.
MPLS TE brings explicit routing capabilities to MPLS networks. An originating label switching route (LSR) can set up a TE label switched path (LSP) to a terminating LSR through an explicitly defined path containing a list of intermediate LSRs. IP uses destination-based routing and does not provide a general and scalable method for explicitly routing traffic. In contrast, MPLS networks can support destination-based and explicit routing simultaneously. MPLS TE uses extensions to RSVP and the MPLS forwarding paradigm to provide explicit routing. These enhancements provide a level of routing control that makes MPLS suitable for TE.

\section{E. Problem Context And Enhacem Ent Of Mpls-Te}

We propose an improvement for the FEC group treatment in order to consider the throughput as an important parameter for multimedia applications that allows it to select the best paths in its routing.

MPLS-TE determines LSP as a sequence of labels in the packet to construct a path and to convey through these paths established by the protocol for distributing labels. The problem of MPLS-TE is how to select the FEC groups that satisfy some parameters of quality of service and in particular the throughput which can be considered as important parameter for some types of applications. The choice of FEC group is also according to several parameters (source address, destination address, QoS parameters). To solve this problem, we group all packets for multimedia applications in a specific FEC, with a high throughput, and LSP that consists of a sequence of labels for multimedia packets is associated with this FEC by the LDP protocol which provides this information to routers LSR on the throughput which we have chosen for packets multimedia in a specified FEC. Hence these multimedia packets take the paths that correspond to throughput as an important quality of service parameter. After the improvement in the FEC group associated with multimedia packets which are labeled, these packets with the principle of MPLS-TE are switched toward the MPLS-TE network by using number of label and the LSP paths. The LSR routers of MPLS-TE network switches the FEC labels that we improved to LER routers, taking into account the throughput that we have set for these multimedia packets.

\section{F. Simulation And Analysis Of The Solution}

To analyze the proposed solution and the effectiveness of our suggested enhancement in MPLS-TE, we use an eventdriven network simulator targeted at networking research. The software version used in this paper is ns-2.34 with MPLS Network Simulator (MNS 2.0).

MPLS-TE and EMPL-TE as discussed in the previous sections have several desirable capabilities. However in this paper, the simulation was chosen to demonstrate the ability of EMPLS-TE in providing Traffic Engineering. To demonstrate this capability, the simulations were setup using a normal MPLS, and a normal MPLS with Traffic engineering implemented (MPLS-TE). The results from these simulations are used for the comparison between the three approaches and evaluate our proposed scheme. Both simulations are based on the common topology. 


\section{G. Simulation environment}

The network consists of 90 nodes (in backbone, sources and destination). All links were set up as duplex with $15 \mathrm{~ms}$ delay and using Drop Tail Queuing, which serve packets on a First Come First Serve (FCFS) basis. The simulation time is 200s and the links have a capacity of $1.5 \mathrm{Mbps}$ and the transmitted flux in the network is multimedia.

TABLE I. SiMULATION PARAMETRS

\begin{tabular}{|l|l|}
\hline \multicolumn{1}{|c|}{ Simulation Parameter } & \multicolumn{1}{c|}{ Value } \\
\hline Simulator & NS-2.34 \\
\hline Simulation Time & $200 \mathrm{~s}$ \\
\hline Node Max. IFQ Length & 50 \\
\hline Data Packet Size & 512 bytes \\
\hline Traffic type & $\mathrm{CBR}(\mathrm{UDP})$ \\
\hline Packet rate & $4 \mathrm{pkt} / \mathrm{sec}$ \\
\hline
\end{tabular}

\section{H. Performance Metrics}

The following metrics are used in varying scenarios to evaluate different protocols:

Packet delivery ratio - This is defined as the ratio of the number of data packets received by the destinations to those sent by the CBR sources.

End-to-end delay of data packets - This is defined as the delay between the time at which the data packet was originated at the source and the time it reaches the destination. Data packets that get lost en route are not considered. Delays due to route discovery, queuing and retransmissions are included in the delay metric.

The metrics are measured against various mobility scenarios and with varying number of data connections.

\section{Comparison between MPLS, MPLS-TE and EMPLS-TE}

In this subsection, we present a comparative analysis of the performance metrics of the MPLS, MPLS-TE and our approach EMPLS-TE.

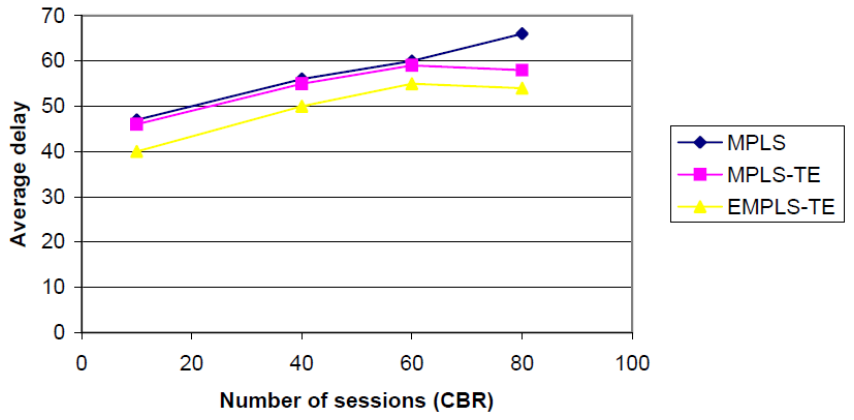

Figure 2. Average delay Vs number of sessions CBR

Packet Delivery Ratio:

Figure 3 gives the packet delivery ratio when the number of sessions (CBR) varies. With number of sessions from 60 to 80 both EMPLS-TE and MPLS-TE has almost same packet delivery ratio but as with number of sessions from 10 to 60 the packet delivery fraction of EMPLS-TE is better. The ratio decreases rapidly in case of MPLS whereas MPLS-TE maintains the same ratio. Thus with the increase in number of sessions EMPLS-TE gives more packet delivery fraction thereby outperforming MPLS and MPLS-TE.

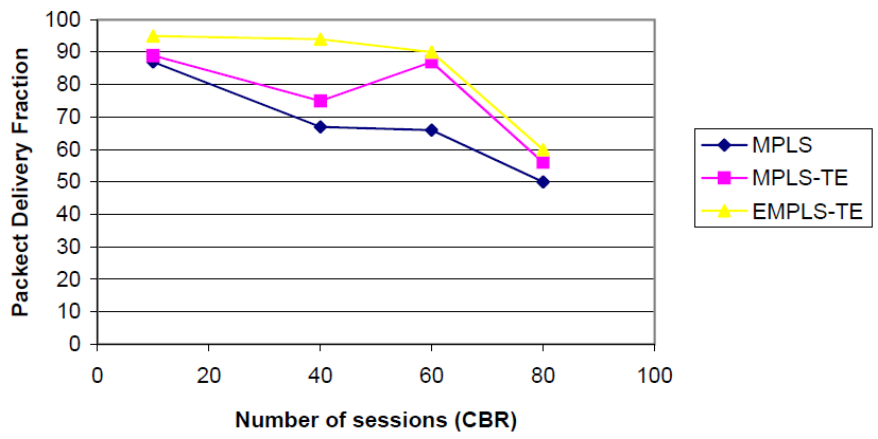

Figure 3. Packet delivery fraction Vs number of sessions CBR

Packets loss :

EMPLS-TE has less packets loss than both MPLS and MPLS-TE under almost all possible values of number of sessions. The difference is magnified under high number of sessions (40 and 60). The primary reason is that in MPLS-TE architecture, we group all packets for multimedia applications in a specific FEC, with a high throughput as compared to that in MPLS. MPLS-TE performs considerably better than both MPLS and MPLS-TE, because MPLS and MPLS-TE focus on LSP routes with the fewest hops, while MPLS-TE tends to choose the least congested route with a specific FEC. Also, when utilizing promiscuous listening MPLS-TE has to spend time processing any control packet it receives, even if it is not the intended recipient. For the time of simulation, the packets loss increases with an increase in the number of sessions.

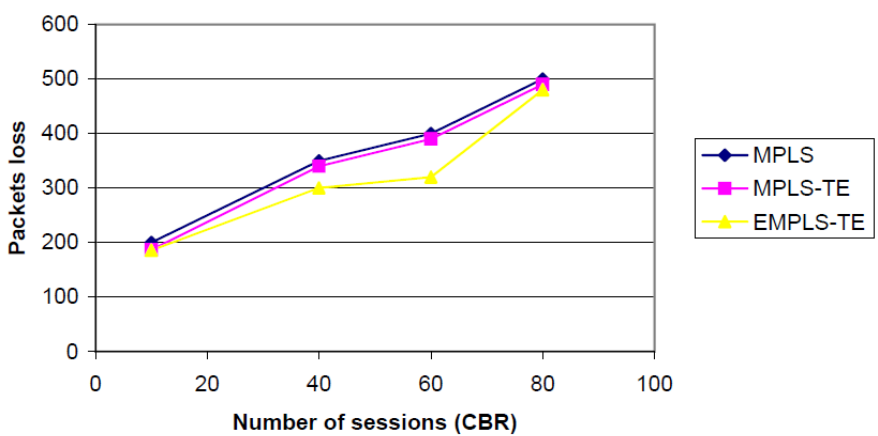

Figure 4. Packets Vs Number of sessions

Throughput with UDP :

From Figure 5, it is clear that at 10 second, MPLS-TE gives better throughput than EMPLS-TE. As the simulation times increases to 50, both MPLS and MPLS-TE have almost the same throughput but as the simulation times increases beyond 200 EMPLS-TE outperforms MPLS-TE and MPLS (as the throughput of all MPLS, MPLS-TE and EMPLS-TE increase with simulation times).

The throughput of EMLPS-TE is similar to MPLS and MPLS-TE between 10s and 50s. The architecture suffers a 
little at fewer simulation times. At low simulation time, the throughput does not exceed 0, 4 Mbps in MPLS, MPLS-TE and EMPLS-TE due to packet collisions. This is because the number of collisions increases in EMPLS-TE due to the additional pending data packets sent by the intermediate routes during route discovery. The throughput increases quickly with increase in simulation times from 100s. Our EMPL-TE solution is very efficient at 200 s. The obtained results show that EMPL-TE is an architecture designed for long periods.

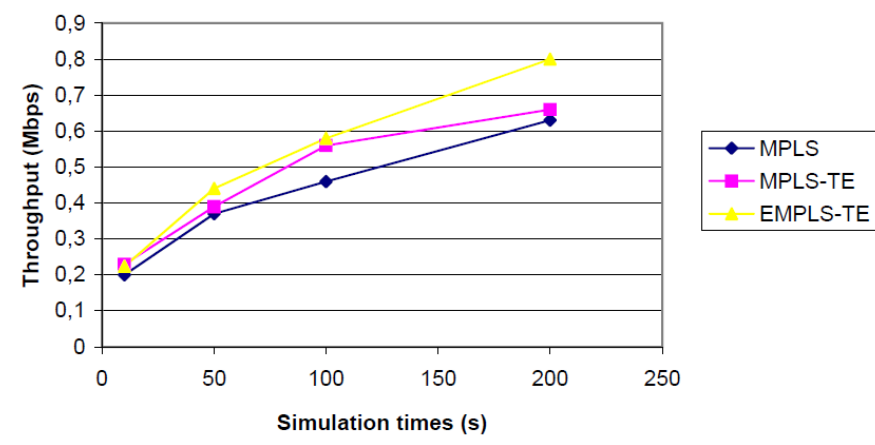

Figure 5. Throughput Vs Simulation times

\section{Throughput with TCP :}

In the first two source nodes send the multimedia stream to their destination through the MPLS network, MPLS-TE network and EMPLS-TE network. And we calculate the TCP throughput and UDP throughput at two destinations (see Figure. 5 and Figure. 6), we note that between 0 second and 10 seconds, our approach EMPLS-TE is more efficient, we obtain an important value of packet delivery ratio, but with author approach (MPLS-TE), the result is not efficient between 0s and $10 \mathrm{~s}$.

This improvement of the packet delivery ratio is due to enhanced throughput with FEC that we changed in MPLS-TE, and as result it performs the transmission of multimedia stream.

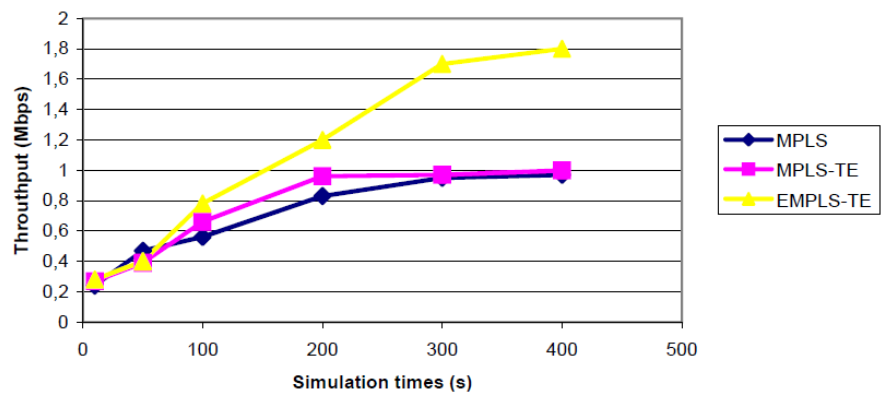

Figure 6. Throughput Vs Simulation times

\section{Conclusion}

Through simulation results and analysis, it was clear that MPLS-TE does not provide a reliable service and improved packet delivery ratio as an important performance metric to ensure the arrival of received packets for sensitive applications as multimedia packets.

\section{ACKNOWLEDGMENT}

This work was supported in part by the networks laboratory at Institute of the Post and TelecommunicationsRabat. We would like to thank for his help and the anonymous referees for their valuable comments.

\section{REFERENCES}

[1] A. Jamali, N. Naja, D. El Oudghiri, and R. Benaini, "Improving quality of service (QoS) in multi-protocol label switching module," in MMS'2009 IEEE Xplore.

[2] R. Callon, E. Rosen, and A. Viswanathan, "Multiprotocol label switching architecture," In IETF RFC3031, Janvier, 2001.

[3] B. Jamoussi, and al. "Constraint based isp setup using idp," In IETF RFC3212, Junuary 2002 ggdd

[4] D. Awduche and al. "Rsvp-te : extensions to rsvp for isp tunnels," In IETF RFC3209, December 2001.

[5] W. Lai, "Requirements for support of differentiated services-aware mpls traffic engineering," In IETF RFC3564, July 2003.

[6] A. Lund, C. Reingold, N. Rexford, J. Feldmann, and A. Greenberg, "Traffic engineering for ip networks," IEEE Network Magazine, vol. 14, pp. 11-19, April 2000.

[7] C. L. Hedrick, "An introduction to igrp," In cisco white paper, August 1991.

[8] H. Wang, J. Liebeherr, J. Wang, and S. Patek, "Traffic engineering with aimd in mpls networks," In LNCS, May 2002.

[9] A. Toguyni, A. Rahmani, K. Lee, and A. Noce, "Comparison of multipath algorithms for load balancing in a mpls network," ICOIN'05, Published by Springer as Lecture Notes in Computer Science (LNCS), pp. 463-470, February 2005.

[10] L. Kyeongja, "Global model for quality of service in fai networks : integration of diffserv and traffic engineering based on mpls," In Thesis, order number 0025. university of science and technology , Central School .

[11] A. Martey, "Integrated is-is routing protocol concepts," In Cisco Press, May 2002.

[12] K. A. M. Lutfullah, M. Z. Hassan, M. R. Amin, M. Arifur Rahman, and A. H. Kabir, "Performance analysis and the study of the be- havior of mpls protocols," In International Conference on Computer and Communication Engineering, pp. 13-15, Kuala Lumpur, Malaysia, May 2008.

[13] P. Lewis James, “Cisco tcp/ip routing professional reference,"revised and expanded. 1998.

[14] S. Berson, S. Herzog, S. Jamin, R. Braden, and L. Zhang, "Resource reservation protocol (rsvp)," In RFC2205, Septembre 1997.

[15] D. Williams R. Guerin, and A. Orda, "Qos routing mechanisms and ospf extensions," In of the Global Internet Miniconference, 1997.

\section{AUTHORS PROFILE}

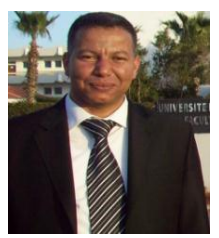

Jamali Abdellah is a researsh and professor at Hassan $1^{\text {er }}$ University, Settat, Morroco, since Ocyober 2011. He was born in Ouarzazate, Morocco. He received a thesis in Computer Science from the University of Hassan II, Mohammedia, Morocco. He is a founding member, in 2007, of a research group e-NGN (e-Next Generation Networks) for Africa and Middle East. His research interests include the computer networks, IPv6 Networks, Quality of Service in MPLS and QoS in Ad Hoc and Networks performance analysis.

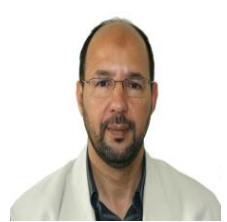

Najib NAJA was born in Maohammadia, Morroco. In 1989, He received his engineering degree from TELECOM Bretagne, France, option : Computer Science and Networks. In 1994, he received the PhD thesis in Computer Science from the University of Rennes I, France. In 1997, he received a thesis in telecommunications from the University of Mohammed V, Morocco. His research interests include the Information 
system, Ad Hoc Network, signal processing, information technology, QoS in Ad Hoc, Networks performance analysis. He is Professor in The National Institute of Posts and Telecommunications (INPT) in Rabat, Morocco since 1994.

Driss El Ouadghiri is a research and an associate professor at Science Faculty, Moulay Ismail University, Meknes, Morocco, since September 1994. $\mathrm{He}$ was born in Ouarzazate, Morocco. He got his "License" in applied mathematics and his "Doctorat de Spécialité de Troisième Cycle" in computer networks, respectively, in 1992 and 1997 from Mohamed V

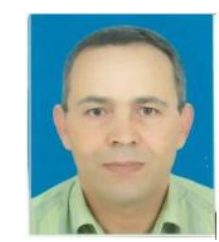

University, Rabat, Morocco. In 2000 he got his PhD in performance evaluation in wide area networks from Moulay Ismail University, Meknes, Morocco. He is a founding member, in 2007, of a research group e-NGN (e-Next Generation Networks) for Africa and Middle East. His research interests focus on performance evaluation in networks (modelling and simulation), DiffServ architecture (mechanisms based active queue management) and IPv6 networks. 